\title{
Improving The Accuracy Rate Of Self-care Of The Oral Cavity In Patients After Head And Neck Tumor Surgery
}

\author{
Wan-Ju Liao, Wei-Hua Yu
}

Department of Nursing, Shin Kong Wu Ho-Su Memorial Hospital, Taipei, Taiwan

\section{Background}

The Head and neck cancer postoperative oral care is pivotal in these patients, as it decreases infection, promotes comfort and prevents complications, and importantly it is one of the factors that enhances the success rate of the surgery. In 2015, a total of 5 patients were dismissed from this ward after being educated for oral care at home. However, these patients were found to have a poor buccal condition later in the outpatient clinic when they came back for a follow-up for which they were asked to be re-educated. An investigation was undertaken and it found that the correctness for self-oral care was only $63.4 \%$, the satisfaction of patients towards the instructions for the oral care given by the nursing staff was only $80.3 \%$.

\section{Methods}

Through a team meeting and using a cause and effect analysis, the following causes and strategies for improving the current situation were emphasized: (1) no standard guidelines for oral care, (2) inefficient and inappropriate guiding material, (3) lack of tracking and consultation system.

Measures for improvement were started in January 2016 which included (Pic 1):

1. Establishing a standard guideline for oral care after head and neck cancer surgery, combining the postoperative management of the indwelling tubings and timing for physical activities, categorizing the steps inthe nursing care of the oral cavity.

2. Creating leaflets and manuals for post-operative care of head and neck cancer surgery, presented in a simple and easy-to-understand text and graphics.

3. Designing a checklist of appropriate oral care instruments which the patients can tick off according to their personal needs.

4. Recording the oral care instructions in multimedia format and corresponding QR code easily accessible through mobile devises.

5. Designing patient self-care progress sheet where the patients and their medical staff can objectively evaluate the patients' conditions and fulfill the standards of the guidance.

6. Designing the contents and schedule for phone calls to the patient for follow-up on the $3^{\text {rd }}$ day after discharge, and evaluation by the attending physician in the outpatient clinic on the $7^{\text {th }}$ day, and subsequent follow-ups through phone calls.

7. Implementing patient self-care progress sheet and corresponding audit to identify particular problems and providing corresponding solutions.

8. Conducting regularly phone interviews and follow-ups and building a data base on the analysis of the results.

\section{Results}

After the implementation of the above-mentionedmeasures, the correct rate of self-oral care for patients with head and neck cancers was remarkably increased from $63.4 \%$ to $90.2 \%$ (Table 1). The satisfaction of patients towards the instructions for the oral care given by the nursing staff was increased from $80.3 \%$ to $94.8 \%$ as well (Table 2), which reflected that the improvement strategy of this project had effective results.

\section{Conclusions}

The design of leaflets, manual and multimedia material for oral care after head and neck cancer not only enhanced the convenience in the instructions by the nursing staff but also greatly improved the learning efficiency and correctness by the patients and their primary caregivers. The statistics resulting from the regular follow-ups of the patients using phone calls for 3 months showed no more patient was referred back for re-education from the outpatient clinic due to poor oral conditions, thus achieving the goal that the patients could take care of themselves correctly on their own, promoting comfort and well-being, decreasing infections and re-hospitalization, and enhancing the patients awareness for a continuous correct self-care of the oralcavity. All these outcomes ultimately highlighted the greatest values of nursing care and favorable outcomes for both the nursing team and patients.

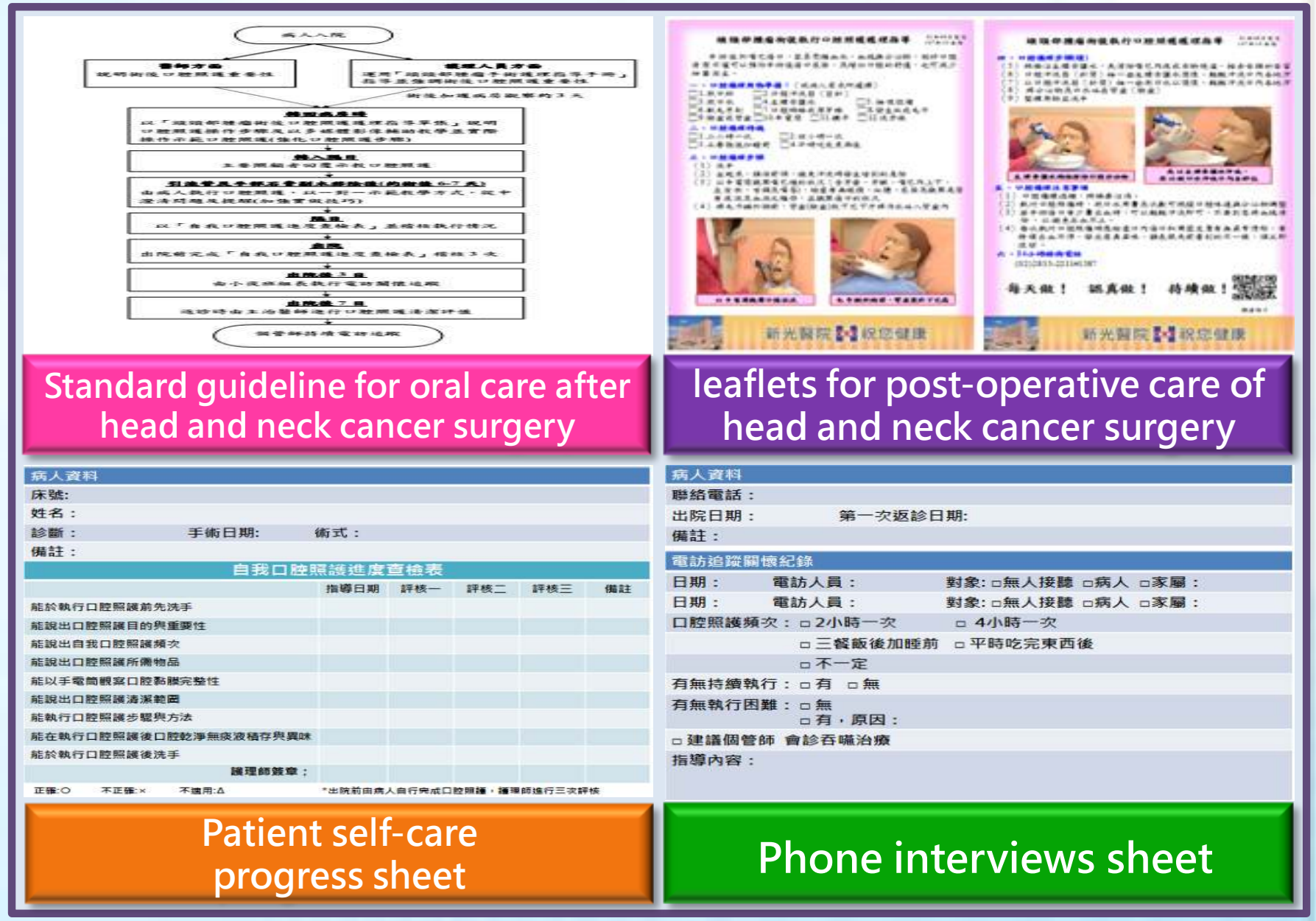

Pic 1: Improve methods

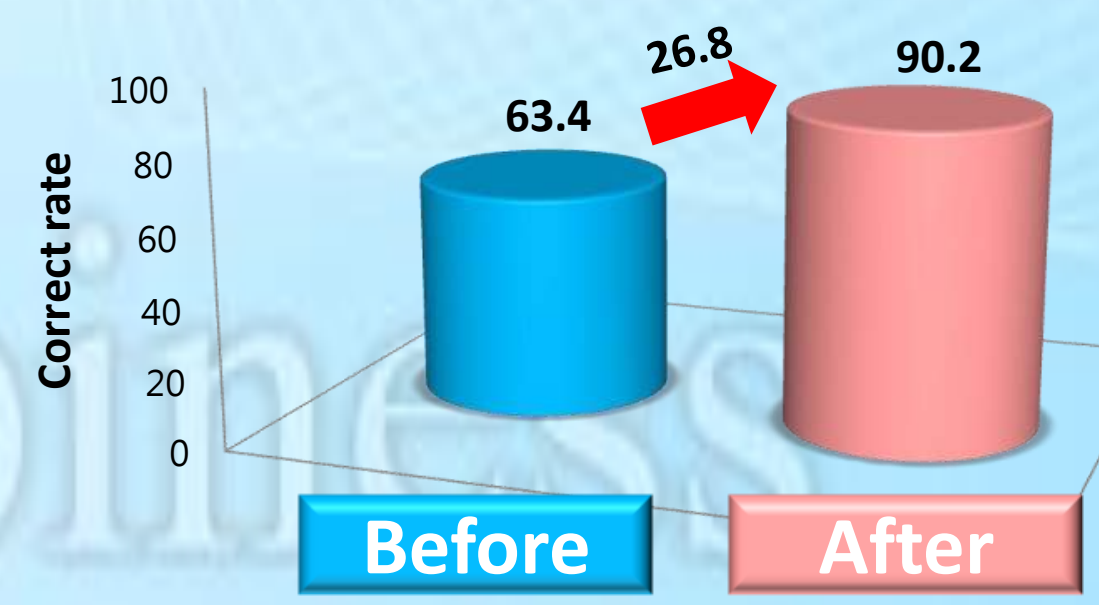

Table 1: The correct rate of self-oral care for patients with head and neck cancer

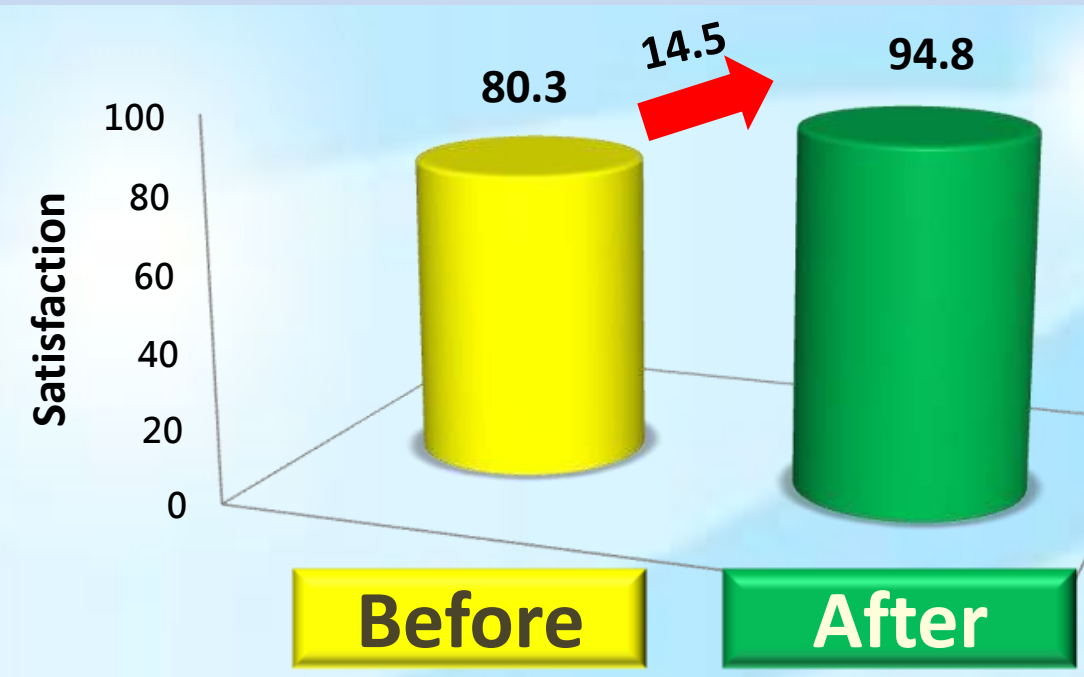

Table 2: The satisfaction of patients towards the instructions for the oral care given by the nursing staff 